\title{
MODELADO Y OPTIMIZACIÓN DE MISIONES DE BÚSQUEDA DE OBJETIVOS MEDIANTE UAVs
}

\author{
Sara Pérez-Carabaza, Eva Besada-Portas, José Antonio López-Orozco \\ Departamento de Arquitectura de Computadores y Automática. Universidad Complutense de Madrid (UCM) \\ sapere04@ucm.es, ebesada@ucm.es, jalo@ucm.es \\ Gemma Blasco \\ Airbus Defence \& Space, Getafe, Madrid \\ gemma.blasco@airbus.com
}

\begin{abstract}
Resumen
La búsqueda de objetivos desde uno o varios vehículos aéreos no tripulados es un problema cuyas aplicaciones abarcan desde la localización de blancos militares a misiones de búsqueda y rescate tras desastres naturales. El tiempo de búsqueda puede ser minimizado mediante un algoritmo que determine las rutas de los vehículos que minimizan el tiempo esperado de localización del objetivo, teniendo en cuenta la información e incertidumbre asociada a cada misión y escenario de búsqueda. Aun más, la idoneidad de las rutas propuestas por estos algoritmos depende tanto de las técnicas de optimización utilizadas como de la adecuación de los modelos a la información disponible e incertidumbre existente. Por tanto, este artículo aborda el problema de forma global, desde la fase de modelado del escenario de búsqueda hasta la optimización de las trayectorias con algoritmos basados en metaheurísticas evolutivas.
\end{abstract}

Palabras clave: Búsqueda en Tiempo Mínimo, Planificación con Incertidumbre, UAVs, Algoritmos Genéticos.

\section{INTRODUCCIÓN}

El problema de búsqueda en tiempo mínimo (MTS, Minimum Time Search) consiste en localizar en el menor tiempo posible un objetivo situado en una posición incierta dentro de un área de búsqueda. Las primeras investigaciones sobre el problema tienen sus orígenes en la Segunda Guerra Mundial y se centran en los problemas de búsqueda de objetivos militares [5]. Su interés ha aumentado durante los últimos años con el desarrollo de los vehículos aéreos no tripulados (UAVs, Unmanned Aerial Vehicles), debido a sus características, especialmente útiles en misiones arriesgadas.

Debido a las múltiples fuentes de incertidumbre asociadas al problema, éste suele abordarse des- de un enfoque probabilístico. La información de la posición del objetivo se modela con un mapa de probabilidad $P\left(\nu^{0}\right)$, donde $\nu^{0}$ es una variable aleatoria que describe la posición inicial del objetivo. Además, en el caso de que el objetivo sea dinámico, el modelo Markoviano $P\left(\nu^{t} \mid \nu^{t-1}\right)$ permite describir de forma probabilística la información relativa a sus movimientos entre dos instantes temporales sucesivos. La correcta definición de ambos modelos es crucial para determinar, a lo largo del tiempo del desarrollo de la misión y con la ayuda de un filtro recursivo bayesiano [1, la probabilidad de encontrar el objetivo en diferentes localizaciones del área de búsqueda. Por ese motivo, en este artículo se muestra un método para la construcción de cada modelo a partir de información del entorno y de la proporcionada por un operador.

Por otro lado, el modelo probabilístico del sensor $P\left(z_{u}^{t} \mid \nu^{t}, s_{u}^{t}\right)$ permite describir la incertidumbre relativa a las medidas $z_{u}^{t}$ del sensor $u$ dada la posición $\nu^{t}$ del objetivo y el estado $s_{u}^{t}$ del UAV y de su sensor. Además, cuando el sensor es una cámara, esta proyecta una huella trapezoidal sobre la zona de búsqueda, fuera de la cual la probabilidad de detectar el objetivo es nula. La incorporación de este modelo en el filtro recursivo bayesiano permite que la trayectoria del UAV y de la huella de su sensor alteren el mapa de probabilidad del objetivo. Por este motivo, este trabajo muestra un algoritmo para optimizar la trayectoria del UAV y la orientación del sensor que incrementa la probabilidad de detectar al objetivo lo antes posible.

Para ilustrar mejor los elementos del problema MTS, la Figura 1 muestra un escenario de búsqueda con un único UAV. El mapa de probabilidad $P\left(\nu^{0}\right)$ representado en la capa superior en escala de grises indica que las zonas con elevaciones más bajas, correspondientes a los valles en verde en el mapa de elevación representado en la capa inferior, tienen mayor probabilidad, representado en tonos más oscuros, de que se encuentre en ellas el objetivo. Además, las flechas azules indican las direcciones más probables de movimiento del ob- 


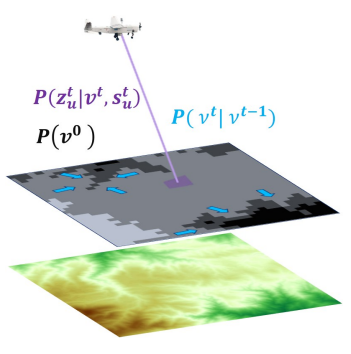

Figura 1: Modelado de las fuentes de incertidumbre de una misión de búsqueda con UAVs.

jetivo, que en este ejemplo hace que el objetivo se mueva desde las zonas de mayor elevación hacia los valles. Finalmente, el trapezoide morado muestra la huella del sensor óptico utilizado para la búsqueda y, por lo tanto, determina la región de observación con probabilidad de detección del objetivo no nula desde la posición actual del UAV.

\section{CONTEXTO}

En el estado del arte actual pueden encontrarse una amplia variedad de trabajos que proponen diferentes métodos de optimización de las rutas de búsqueda de uno 6, 13 o varios UAVs 7, 9, 11 de acuerdo a alguna función objetivo que tiene en cuenta la incertidumbre del escenario, modelada mediante $P\left(\nu^{0}\right), P\left(\nu^{t} \mid \nu^{t-1}\right)$ y $P\left(z_{u}^{t} \mid \nu^{t}, s_{u}^{t}\right)$. Aunque es relativamente habitual optimizar la probabilidad de encontrar el objetivo [13] o la entropía [16], en este trabajo en el que se afronta el problema MTS, optimizaremos el valor esperado de encontrar el objetivo ya que es el criterio más adecuado para este problema 6, 7, 9, 11]. Además, debido a la NP-complejidad del problema de búsqueda 15, éste suele abordarse con métodos de optimización aproximados o metaheurísticas (p.e. mediante algoritmos de optimización de entropía cruzada [6], colonias de hormigas [9], o genéticos [1]) que no garantizan la obtención de la solución óptima. Ejemplos adicionales de funciones objetivo y algoritmos usados en problemas de búsqueda pueden encontrase en las revisiones bibliográficas de los artículos anteriores y en [14].

Las técnicas de modelado y optimización descritas en este artículo, y detalladas más extensamente en [12, forman parte del proyecto de innovación abierta SAVIER [4], mediante el que Airbus ha financiado 12 líneas de investigación relacionadas con las futuras generaciones de sus estaciones de tierra (Ground Control Station, GCS) en 5 universidades españolas. Los objetivos industriales de este trabajo son proporcionar al operador de la GCS herramientas que 1) faciliten la definición de misiones de búsqueda realistas y 2) automaticen la pre-planificación de las trayectorias de sus UAVs.
Finalmente, cabe destacar que ambos objetivos son igualmente importantes, ya que para que un operador pueda utilizar los resultados del planificador, también debe disponer de herramientas que le permitan definir ágilmente las misiones. Por eso, este artículo 1) recoge la metodología desarrollada para definir y modelar la misión de búsqueda y, 2) describe las características más relevantes del algoritmo desarrollado para la GCS de Airbus y los resultados obtenidos sobre una misión realista.

\section{MODELOS DEL PROBLEMA}

En esta sección se describen los métodos propuestos en 12] para ayudar al operador a modelar probabilísticamente la localización inicial y el movimiento del objetivo, y las funciones utilizadas para modelar la probabilidad de detección del sensor y el movimiento determinista de los UAVs.

\subsection{MODELO PROBABILÍSTICO DE LA POSICIÓN INICIAL DEL OBJETIVO}

Para definir el mapa de probabilidad inicial $b\left(\nu^{0}\right) \equiv P\left(\nu^{0}\right)$ se discretiza uniformemente el área de búsqueda rectangular $\Omega$, quedando ésta dividida en una red $G_{\Omega}$ de $w_{x} \cdot w_{y}$ celdas. Una vez discretizada $\Omega$, se asigna a cada celda del mapa una probabilidad de que el objetivo se encuentre en ella. Además, se asume que el objetivo se encuentra dentro de $\Omega$, por lo que $\sum_{\nu^{0} \in G_{\Omega}} b\left(\nu^{0}\right)=1$.

Para asignar dichas probabilidades se propone combinar la información sobre la localización del objetivo procedente de $J$ fuentes de información diferentes mediante la Ecuación 1, donde $b^{j}\left(\nu^{0}\right)$ es la capa de probabilidad de la fuente de información $j$ y $w_{j}$ el peso que el operador asigna a dicha fuente de información de acuerdo a su fiabilidad.

$$
b\left(\nu^{0}\right)=\frac{1}{\sum_{j=1}^{J} w_{j}} \sum_{j=1}^{J} w_{j} \cdot b^{j}\left(\nu^{0}\right)
$$

Más concretamente en este artículo utilizamos las dos fuentes de información consideradas en SAVIER: $b^{1}\left(\nu^{0}\right)$ recoge la información asociada a la localización inicial del objetivo según la elevación del terreno y $b^{2}\left(\nu^{0}\right)$ la proporcionada, para cada misión concreta, por los servicios de emergencia.

Más en detalle, para definir $b^{1}\left(\nu^{0}\right)$ se utiliza un modelo digital de la elevación (DEM, Digital Elevation Model, [8]) del área de búsqueda $\Omega$ que es remuestreado automáticamente al tamaño de celdas de $G_{\Omega}$. A continuación el operador solo debe definir los rangos de elevación en los que desea dividir $\Omega$ y asignar las probabilidades de que el objetivo se encuentre en ellos. Con esta información, la herramienta determina automáticamente 
el rango de elevación al que pertenece cada celda $\nu^{0} \in G_{\Omega}$ y le asigna la $b^{1}\left(\nu^{0}\right)$ que le corresponde.

Por otro lado, para obtener $b^{2}\left(\nu^{0}\right)$, el operador puede seleccionar gráficamente las últimas localizaciones conocidas del objetivo y su posible dispersión, o las regiones poligonales sobre las que puede encontrarse. El operador también debe indicar la importancia que da a cada una de estas fuentes de información. A continuación, la herramienta obtiene $b^{2}\left(\nu^{0}\right)$ de forma automática, considerando que el operador ha definido una mezcla de gausianas centradas en las últimas posiciones conocidas y de uniformes sobre las regiones poligonales.

Finalmente, el operador también debe elegir el valor de los pesos $w_{1}$ y $w_{2}$ que se utilizarán para combinar la probabilidad relativa a la elevación del terreno y a los servicios de emergencia. También debe comprobar visualmente que el resultado $b\left(\nu^{0}\right)$ refleja la probabilidad inicial de la misión, ya que de no ser así, los resultados obtenidos por el planificador no serán adecuados para la misión.

\subsection{MODELO PROBABILÍSTICO DEL MOVIMIENTO DEL OBJETIVO}

Para construir el modelo probabilístico de la dinámica del objetivo, el operador define las probabilidades de que desde un subconjunto de las celdas de $G_{\Omega}$ el objetivo se mueva a sus celdas adyacentes, y el método propuesto extiende de forma automática la dinámica definida por el operador al resto de celdas del área de búsqueda.

En mayor detalle, el operador proporciona, a través de una herramienta gráfica, la información de $K$ vectores $v_{1: K}$ relativos a $\mathrm{K}$ celdas $c_{k} \in G_{\Omega}$ elegidas por él. En cada vector $v_{k}$ debe definir la dinámica del objetivo relativa a la celda $c_{k}$ de la siguiente manera: los 8 primeros elementos de $v_{k}$ se corresponden con las probabilidades de que el objetivo se desplace en dos instantes temporales consecutivos de $c_{k}$ a cada una de las celdas adyacentes siguiendo las 8 direcciones cardinales $d \in\{1: 8\}$ y el noveno elemento de $v_{k}$ indica la probabilidad de que el objetivo permanezca fijo en $c_{k}$.

A continuación, el método propuesto para la definición de $P\left(\nu^{t} \mid \nu^{t-1}\right)$ extiende automáticamente, mediante la Ecuación 2, la dinámica definida por $v_{1: K}$ en $K$ celdas del mapa al resto de celdas de $G_{\Omega}$. Dicha ecuación obtiene la probabilidad, de que el objetivo se mueva de la celda $\nu^{t-1}$ a la celda adyacente $\nu^{t}=\operatorname{adj}\left(\nu^{t-1}, d\right)$ siguiendo la dirección cardinal $d$, ponderando el valor $v_{k}[d]$ de los K vectores con la distancia entre la celda $\nu^{t}$ (destino del objetivo) y $c_{k}$ (correspondiente al vector $v_{k}$ ). Por último, $\eta$ es un factor de normalización que asegura la suma unitaria de la probabilidad de que el objetivo se desplace hacia sus celdas adyacentes o permanezca fijo en la misma posición.

$$
P\left(\nu^{t}=\operatorname{adj}\left(\nu^{t-1}, d\right) \mid \nu^{t-1}\right)=\frac{1}{\eta} \sum_{k=1: K} \frac{v_{k}[d]}{\operatorname{dist}\left(\nu^{t}, c_{k}\right)}
$$

Finalmente, para que el operador pueda comprobar si la dinámica del objetivo que ha definido es correcta, la herramienta 1) representa las direcciones cardinales más probables sobre cada celda del mapa y 2) le permite observar como la dinámica seleccionada modifica $b\left(\nu^{t}\right)$ a lo largo de tiempo.

\subsection{MODELO PROBABILÍSTICO DEL SENSOR}

El modelo del sensor $P\left(z_{u}^{t} \mid \nu^{t}, s_{u}^{t}\right)$ define la probabilidad de obtener una medida $z_{u}^{t}$ por el UAV $u$ en el instante de tiempo $t$ en función de la posición del objetivo $\nu^{t}$ y del estado del UAV $s_{u}^{t}$.

En la fase final de SAVIER, se utiliza una cámara modelada con la Ecuación 3, donde $\left|\nu^{t}\right|$ es el área total de la celda, $\left|\nu^{t} \cap h u e l l a\left(s_{u}^{t}\right)\right|$ representa el área de la región común entre la celda y la huella del sensor huella $\left(s_{u}^{t}\right)$ y $P_{\text {camara }}$ la probabilidad de detección, es decir de que $z_{u}^{t}=D$, de la cámara elegida. Por lo tanto, el modelo pondera la probabilidad $P_{\text {camara }}$ mediante un factor proporcional al área de la celda contenida en la huella del sensor. Además, la huella del sensor huella $\left(s_{u}^{t}\right)$ es obtenida considerando la posición del UAV $\left(x_{u}^{t}, y_{u}^{t}, h_{u}^{t}\right)$, la altitud media del terreno, y los ángulos de elevación $\gamma$ y azimut $\alpha$ de la cámara.

$$
P\left(z_{u}^{t}=D \mid \nu^{t}, s_{u}^{t}\right)=\frac{\mid \nu^{t} \cap \text { huella }\left(s_{u}^{t}\right) \mid}{\left|\nu^{t}\right|} P_{\text {camara }}
$$

Para poder utilizar este modelo el operador únicamente debe pre-estimar el valor de la constante $P_{\text {camara }}$, teniendo en cuenta diversos factores como el tamaño del objetivo, la resolución de la cámara, las condiciones meteorológicas, etc., y siguiendo los procedimientos habituales en la definición de las misiones de búsqueda.

\subsection{MODELO DINÁMICO DEL UAV}

El modelo determinista de la dinámica de los UAVs $s_{u}^{t}=f\left(s_{u}^{t-1}, a_{u}^{t-1}\right)$ permite actualizar el estado $s_{u}^{t}$ del UAV a partir de su estado en el instante anterior $s_{u}^{t-1}$ y las consignas $a_{u}^{t-1}$.

Para agilizar el proceso de planificación, en este artículo se considera un modelo dinámico no lineal implementado en Simulink, que captura la dinámica de alto nivel de un UAV de ala fija y que ha sido utilizado previamente en [9, 11]. En la Figura 2 se resalta con colores las distintas variables del modelo: en agua-marina las consignas 


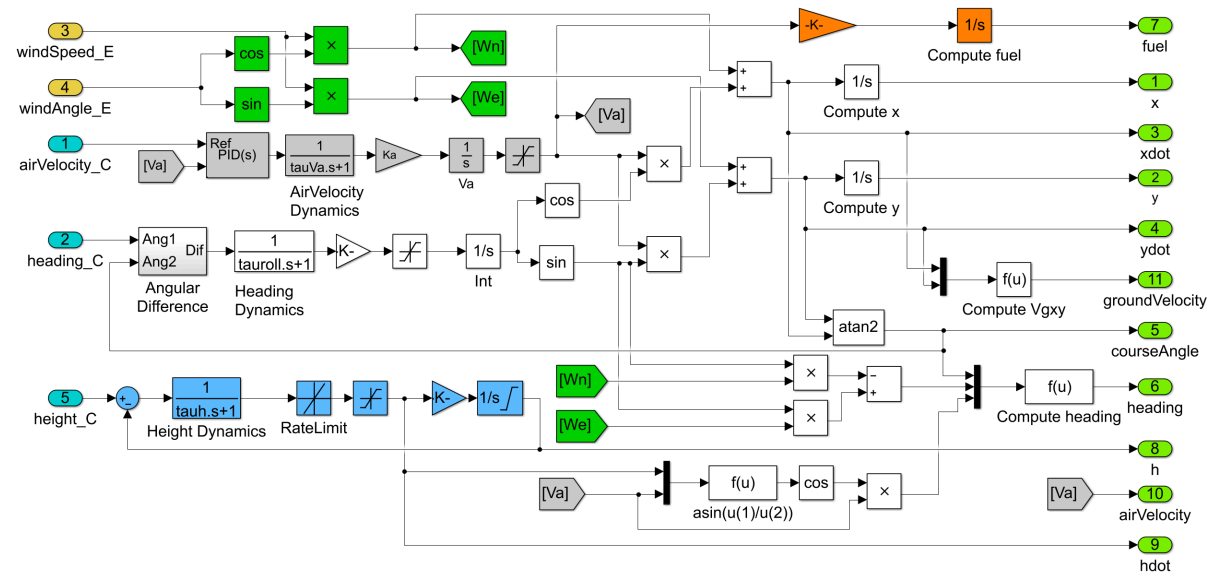

Figura 2: Modelo dinámico de los UAVs implementado en Simulink.

(velocidad, rumbo y altura), en amarillo las perturbaciones (viento) y en verde claro las variables de estado del UAV (posición, altitud, consumo de fuel, ángulos de rumbo y derrota, y velocidades correspondientes). Además, los bloques naranja resaltan la dinámica del fuel, los grises la de velocidad, los azules la de altura, los blancos la lateral y los verde la del viento.

Finalmente, conviene indicar que el comportamiento de este modelo está parametrizado en un fichero de configuración, seleccionable antes del comienzo de la misión, que permiten al operador indicar las restricciones aerodinámicos (p.e. ángulo de alabeo, velocidad de ascenso) de cada UAV. De esta forma, se permite que el operador defina de forma independiente el comportamiento de cada UAV y que el planificador utilice un modelo computacionalmente eficiente para simularlos.

\section{OPTIMIZACIÓN DE LAS TRAYECTORIAS}

En esta sección se describe el algoritmo MTS desarrollado en SAVIER y la estrategia seguida para evaluar las trayectorias de búsqueda. Información adicional de ambos puede encontrarse en [12].

\subsection{ALGORITMO DE OPTIMIZACIÓN}

El método de optimización elegido es de la familia de los Algoritmo Genéticos (GA, [3]). Los GA son metaheurísticas ampliamente utilizadas que, mediante operadores de cruce y mutación de inspiración biológica, permiten mejorar iterativamente una población de individuos/soluciones (en nuestro caso trayectorias de búsqueda) seleccionando los individuos en función de su idoneidad (en nuestro caso, el tiempo esperado de detección del objetivo y otros criterios adicionales).
Las soluciones se han codificado en el GA a partir de las consignas. Se ha elegido esta codificación, ya que permite obtener trayectorias coherentes con la restricciones dinámicas definidas por el modelo dinámico $s_{u}^{t}=f\left(s_{u}^{t-1}, a_{u}^{t-1}\right)$ de los UAVs, a partir de la posición inicial del UAV y la secuencias de consignas, cuyos valores serán aplicados de forma constante durante cada intervalo temporal. Concretamente en este artículo, y por limitaciones de espacio, se considera la optimización del ángulo de rumbo $\theta_{u}^{t}$, mientras que la velocidades $v_{u}^{t}$ y altura $h_{u}^{t}$ de los UAVs se consideran constantes. Es decir, el algoritmo MTS propuesto, basado en GA, optimiza la secuencia de consignas del ángulo de rumbo $\theta_{1: U}^{1: N}$ (que constituyen el genotipo del GA) y evalúa las trayectorias de búsqueda $s_{1: U}^{0: N}$ (su fenotipo). Además, en la configuración del GA se utilizan los siguientes operadores: selección mediante un torneo binario, cruce por un punto con probabilidad $p_{x}=0.8$, y la mutación gaussiana de dos niveles definida en [1].

Finalmente, para resolver el problema se considera una estrategia de Control Predictivo por Modelo $(\mathrm{CPM})$, que permite dividir la optimización de la trayectoria completa $s_{1: U}^{0: N}$ de horizonte de decisión $N$ en $R$ tramos consecutivos con un horizonte de optimización menor $L=N / R$. Con esta elección se reduce el espacio de búsqueda del GA y la complejidad computacional de cada iteración del optimizador 11, 12. Para implementar esta estrategia multi-etapa, durante la optimización de cada sub-secuencia de consignas de la trayectoria $\left\{\theta_{1: U}^{1: L}, \ldots, \theta_{1: U}^{l: r L}, \ldots, \theta_{1: U}^{(R-1) L+1: R L}\right\}$ se considera como posición inicial de los UAVs la última posición del tramo anterior $s_{1: U}^{(r-1) L}$ y como mapa de probabilidades inicial la creencia no normalizada actualizada hasta el tramo anterior $\tilde{b}\left(\nu^{(r-1) L}\right)$. Esta creencia sin normalizar es definida en la siguiente sección. Para el caso de la optimización del primer tramo $\left(\theta_{1: U}^{1: L}\right)$ se toman como punto de partida la 
posición inicial de los UAVs $s_{1: U}^{0}$ y el mapa de probabilidad inicial $b\left(\nu^{0}\right)=P\left(\nu^{0}\right)$.

\subsection{CRITERIOS DE EVALUACIÓN}

El planificador propuesto evalúa las trayectorias de los UAVs de acuerdo a una restricción que penaliza las colisiones entre UAVs y a los dos criterios de optimización detallados a continuación.

Penalización de colisiones entre UAVs. Para evaluar este criterio se suman los periodos de tiempo en los que las trayectorias de los UAVs están separadas una distancia inferior al radio de seguridad.

Tiempo esperado de detección. En las misiones MTS hay que determinar las trayectorias de búsqueda de los UAVs que minimizan el tiempo de encontrar el objetivo. Sin embargo, el tiempo exacto de detección no puede ser calculado debido a la incertidumbre asociada al problema. Por ello, habitualmente se minimiza el valor Esperado del Tiempo (ET) de detección [7, 9, 11, 12, que puede ser calculado mediante la Ecuación 4 y el proceso iterativo definido por la Ecuación 5 .

$$
\begin{gathered}
\operatorname{ET}\left(s_{1: U}^{1: N}\right)=\sum_{t=0}^{N} \sum_{\nu^{t} \in G_{\Omega}} \tilde{b}\left(\nu^{t}\right) \cdot \Delta T \\
\tilde{b}\left(\nu^{t}\right)=\prod_{u=1}^{U} P\left(z_{u}^{t}=\bar{D} \mid \nu^{t}, s_{u}^{t}\right) \cdot \\
\sum_{\tau^{t-1} \in \Omega_{G}} P\left(\nu^{t} \mid \nu^{t-1}\right) \tilde{b}\left(\nu^{t-1}\right)
\end{gathered}
$$

donde $\Delta T$ es el intervalo temporal entre dos medidas consecutivas del sensor, y $\tilde{b}\left(\nu^{t}\right)$ es la creencia no normalizada, actualizada con la dinámica del objetivo y la medidas del sensor, de acuerdo a los modelos $P\left(\nu^{t} \mid \nu^{t-1}\right)$ y $P\left(z_{u}^{t}=\bar{D} \mid \nu^{t}, s_{u}^{t}\right)$. $\tilde{b}\left(\nu^{t}\right)$ se obtiene siguiendo el procedimiento demostrado en [7], que es un filtro recursivo bayesiano modificado al no considerar el factor de normalización habitual y asumir que en todo instante se producen medidas de no detección $z=\bar{D}$. Además, como el modelo de detección del sensor únicamente considera que se pueden producir medidas de detección $(D)$ y no detección $(\bar{D})$, $P\left(z_{u}^{t}=\bar{D} \mid \nu^{t}, s_{u}^{t}\right)=1-P\left(z_{u}^{t}=D \mid \nu^{t}, s_{u}^{t}\right)$, siendo este último el modelo usado en la Ecuación 3. Por lo tanto, si el objetivo permanece inmóvil, $b\left(\nu^{t}=c\right)<\tilde{b}\left(\nu^{t-1}=c\right)$ para aquellas celdas $c \in G_{\Omega}$ que en el instante $t$ se encuentran bajo la huella de la cámara (obtenida a partir de la localización del vehículo $\left.s_{u}^{t}\right)$ y $\tilde{b}\left(\nu^{t}=c\right)=\tilde{b}\left(\nu^{t-1}=c\right)$ para aquellas celdas fuera de dicha huella. Es decir, $\tilde{b}\left(\nu^{t}\right)$ disminuye en las celdas localizadas dentro de la huella actual de la cámara, y el movimiento del objetivo hace que $\tilde{b}\left(\nu^{t}\right)$ se redistribuya desde una celda a sus adyacentes.

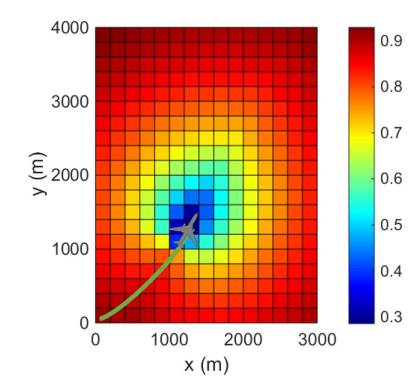

Figura 3: Esquema del criterio de corrección de la miopía.

El tiempo esperado (ET) de detectar el objetivo desde las trayectorias de los UAVs $s_{1: U}^{1: N}$ se obtiene sumando, para cada instante temporal, el valor total de $\tilde{b}\left(\nu^{t}\right)$ sobre todas las celdas de $G_{\Omega}$, multiplicado por el intervalo temporal $\Delta T$. Como acabamos de ver, el valor total de $\tilde{b}\left(\nu^{t}\right)$ decrece según las huellas de las cámaras de los UAVs observan las diferentes celdas del mapa, por lo que $\sum_{\nu^{t} \in \Omega_{G}} \tilde{b}\left(\nu^{t}\right) \leq \sum_{\nu^{t} \in \Omega_{G}} \tilde{b}\left(\nu^{t-1}\right) \leq 1$. Es decir, según los UAVs recorren su trayectoria, el valor que se añade al cómputo del ET es más pequeño, pudiendo ser nulo para aquellos casos en los que la trayectoria de la huella de la cámara hace que $\sum_{\nu^{t} \in \Omega_{G}} \tilde{b}\left(\nu^{t}\right)=0$ para algún $t \leq N$. En esos casos, la Ecuación 4 calcula el valor esperado de detección. Sin embargo, cuando no se cumple la condición anterior, aunque la Ecuación 4 subestima el ET es capaz de determinar las trayectorias mejores respecto a dicho criterio hasta $t=N[6,7]$.

Criterio de corrección de la miopía. Este objetivo disminuye el efecto de miopía derivado de la optimización de la trayectoria completa de búsqueda $s_{1: U}^{1: N}$ en varios tramos $(r=1, \ldots, R)$ de horizonte de decisión $L$, siguiendo una estrategia CPM. El objetivo propuesto (MYOP) en las Ecuaciones 6 y 7. utiliza una heurística $H\left(\nu^{r L}, s_{u}^{r L}\right)$ para ponderar $\tilde{b}\left(\nu^{r L}\right)$ y penalizar las trayectorias de los UAVs cuyas posiciones finales $s_{1: U}^{r L}$ se encuentran a mayor distancia $\operatorname{dist}\left(\nu^{r L}, s_{u}^{r L}\right)$ de las zonas de alta creencia no normalizada de presencia del objetivo.

$$
\begin{gathered}
\text { MYOP }=\sum_{\nu \in G_{\Omega}} \prod_{u=1}^{U} H\left(\nu^{r L}, s_{u}^{r L}\right) \tilde{b}\left(\nu^{r L}\right) \\
H\left(\nu^{r L}, s_{u}^{r L}\right)=1-\lambda^{\operatorname{dist}\left(\nu^{r L}, s_{u}^{r L}\right)} \text { con } 0<\lambda<1
\end{gathered}
$$

En la Figura 3 se representa la función $H\left(\nu^{r L}, s_{u}^{r L}\right)$ correspondiente a un UAV cuya posición final $s_{u}^{r L}$ en el tramo actual de la trayectoria es igual a $(1200,1200) \mathrm{m}$. Al ser MYOP un objetivo que se debe minimizar, $H\left(\nu^{r L}, s_{1}^{r L}\right)$ penaliza las zonas de que se encuentran más alejadas de $s_{1}^{r L}$. De esta manera, MYOP favorece que las posiciones finales de los UAVs queden cerca de las áreas con valores altos de $\tilde{b}\left(\nu^{r L}\right)$. 


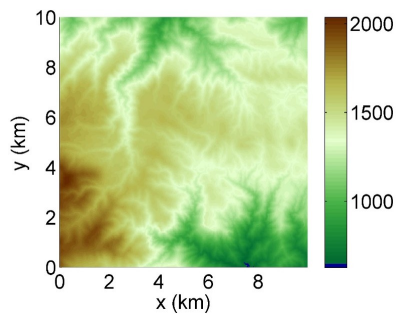

(a) Elevación (m)

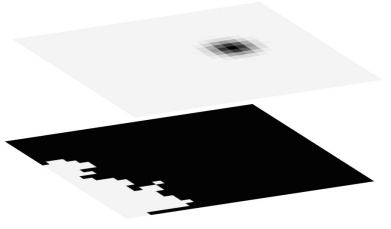

(b) Definición $P\left(\nu^{0}\right)$

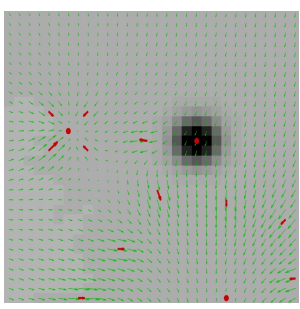

(c) Definición $P\left(\nu^{t} \mid \nu^{t-1}\right)$

Figura 4: Definición de los modelos probabilísticos de un objetivo perdido en la Sierra de Gádor (Almería, España) a partir de la información de elevación del terreno y la información proporcionada por el operador.

Es importante resaltar el carácter complementario de los dos criterios anteriores. Mientras que la minimización del ET favorece que los UAVs sobrevuelen lo antes posible zonas con altos valores de $\tilde{b}\left(\nu^{l}\right)$ alcanzables dentro del horizonte de decisión $L$, la minimización de MYOP favorece que las posiciones finales $s_{1: U}^{r L}$ de cada tramo $r$ sean adecuadas para la optimización de los tramos posteriores.

Para resolver el problema de optimización mutiobjetivo con restricciones planteado se sigue la estrategia propuesta en [2]: se priorizan las soluciones factibles frente a las que no cumplen la restricción, y las soluciones con valores de penalización iguales se ordenan según su ET y MYOP de acuerdo a la estrategia multi-objetivo Pareto del algoritmo NSGA-II. Esta forma de proceder hace que el GA devuelva un frente Pareto de soluciones factibles tras la optimización de cada tramo de la trayectoria, entre las que es necesario elegir automáticamente una, cuya posición final es tomada como punto de partida de la optimización del tramo siguiente. Para hacerlo, tras comprobar las elecciones del operador ante diferentes escenarios y frentes Pareto de soluciones, se ha decidido redondear el valor de MYOP a un decimal y elegir aquella solución con menor MYOP redondeado.

\section{CASO DE USO}

En esta sección se utiliza un escenario realista propuesto por Airbus dentro del proyecto SAVIER para poner a prueba las contribuciones del planificador MTS en sus GCSs. Información sobre el proceso de integración del planificador en la GCS del UAV ATLANTE puede encontrarse en [10.

La misión elegida es realizada por dos UAVs y tiene como objetivo encontrar a un montañero perdido en un área de 10×10 km² situada en la Sierra de Gádor (España).

Para la construcción de $P\left(\nu^{0}\right)$ se discretiza la zona de búsqueda en un enrejado de $30 \times 30$ celdas, y se utilizan una capa de probabilidad construida a partir de la elevación del terreno representado en la Figura 4 (a) y otra a partir de la información de emergencia. En la Figura 4(b) se representan ambas capas de probabilidad, indicando con colores más oscuros las celdas donde es mas probable que se localice el objetivo. La primera capa de probabilidad $b^{1}\left(\nu^{t}\right)$, representada en la parte inferior de la Figura 4 (b), informa de la baja probabilidad de que el montañero se encuentre en elevaciones superiores a 1700 metros. La segunda capa de probabilidad $b^{2}\left(\nu^{t}\right)$, representada en la parte superior de la Figura 4 (b), define una gaussiana con alta probabilidad de localización centrada en la última posición conocida del montañero. El mapa de probabilidades inicial $P\left(\nu^{0}\right)$,representado en la Figura 5 (a), se obtiene fusionando la información de ambas capas de acuerdo a los pesos $w_{1}=0,80 \mathrm{y}$ $w_{2}=0,20$ proporcionados por el operador, que da una importancia 4 veces más alta a la información de la elevación que a la de emergencia.

Además, el montañero se mueve con una velocidad aproximada de $1.38 \mathrm{~m} / \mathrm{s}$ y es posible que se dirija hacia un refugio situado en un pequeño valle al oeste o hacia el sur en dirección al lago que se encuentra a una elevación menor. Esta información se utiliza para construir el modelo dinámico del objetivo $P\left(\nu^{t} \mid \nu^{t-1}\right)$, describiendo la dinámica sobre varias celdas del mapa, cuyas direcciones de movimiento más probables se indican con flechas rojas, sobre $P\left(\nu^{0}\right)$, en la Figura 4 (c). Además, también se considera probable que el montañero permanezca quieto en su última posición conocida, en el refugio o cerca del lago, indicando esta información con los puntos rojos de la Figura 4 (c). A continuación, la herramienta y metodología descritas en la Sección 3.2 extienden la dinámica definida en $c^{k}$ (celdas con flechas o puntos rojos) al resto de celdas $\nu^{t} \in G_{\Omega}$, obteniéndose una $P\left(\nu^{t} \mid \nu^{t-1}\right)$, cuyas direcciones más probables de movimiento se indican con flechas verdes en Figura 4 (c).

La Figura 5 (a) muestra el mapa de probabilidad inicial $P\left(\nu^{0}\right)$ y el punto de entrada, al nordeste de $G_{\Omega}$ y una hora después del momento en que se perdió el rastro del montañero, de los dos UAVs involucrados en la misión. Por lo tanto, el mapa de 


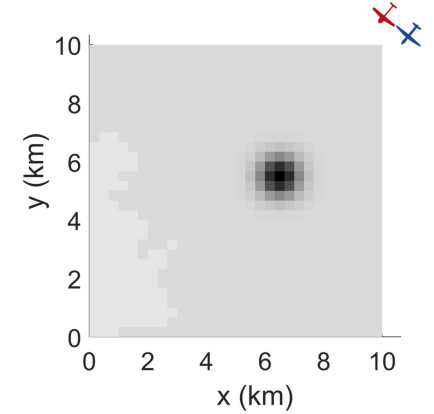

(a) $P\left(\nu^{0}\right)$ del escenario inicial

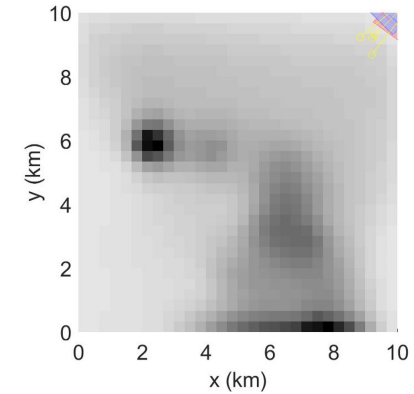

(b) $P\left(\nu^{t}\right)$ al inicio del búsqueda

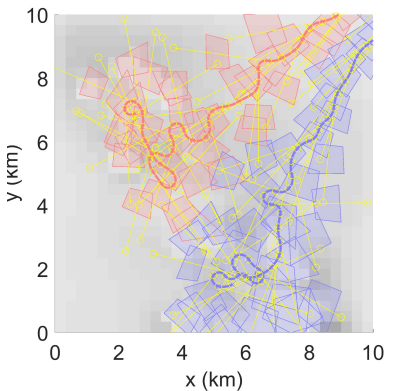

(c) Solución propuesta

Figura 5: Mapa de creencias (sin normalizar) y rutas propuestas por el algoritmo de búsqueda.

probabilidad al inicio de la misión ha cambiado de acuerdo a la dinámica definida por $P\left(\nu^{t} \mid \nu^{t-1}\right)$, obteniéndose la $P\left(\nu^{t}\right)$ que se representa en la Figura 5 (b), y en la que la la probabilidad de localización del montañero se ha desplazado hacia las celdas cercanas al refugio y al lago situado al sur.

Además, para terminar de parametrizar la misión, hay que tener en cuenta que: los UAVs se desplazan con una velocidad y altura constantes $(v=40$ $\mathrm{m} / \mathrm{s}$ y $h=3000 \mathrm{~m}$ ); que la probabilidad de detección del sensor dentro de su huella $P_{\text {camara }}=0.95$ (debido a la altura de vuelo y la altitud media del terreno); que el sensor mantiene una elevación $\left(\gamma=45^{\circ}\right)$ y azimut $\left(\alpha=0^{\circ}\right)$ constantes, y que realiza medidas cada $\Delta T=10 \mathrm{~s}$.

La Figura 5(c) muestra una solución obtenida por el algoritmo propuesto, tras una ejecución de 5 minutos de duración. En la figura, las líneas gruesas representan la trayectoria de los UAVs, los trapezoides las huellas del sensor, y las líneas amarillas relacionan cada huella con el punto de la trayectoria desde el que se realizado la medida. En la gráfica se observa que los dos UAVs se dirigen hacia las zonas con mayor probabilidad de presencia del objetivo perdido: el UAV rojo se desplaza directamente y sobrevuela la zona del refugio, y el UAV azul sobrevuela primero la última posición conocida del objetivo y después se dirige hacia el valle cercano al lago.

Finalmente, hay que tener en cuenta que, debido a la naturaleza estocástica de los GA, las trayectorias propuestas por estos pueden ser diferentes en cada ejecución. Por lo tanto, se ejecutan 20 optimizaciones diferentes y se analizan los resultados. En todas ellas los dos UAVs se distribuyen para poder sobrevolar las zonas de mayor probabilidad (cercanas al refugio, a la ultima posición conocida del montañero y al lago), obteniendo una probabilidad media de encontrar al objetivo al final de la misión de $P_{\text {deteccion }}\left(s_{1: U}^{t: N}\right)=0.77 \pm 0.01 \mathrm{y}$ un tiempo esperado medio de detección del objetivo de $E T\left(s_{1: U}^{t: N}\right)=320 \pm 7 \mathrm{~s}$.

\section{CONCLUSIONES}

Este artículo describe un planificador desarrollado en el proyecto de innovación abierta SAVIER, en el que se exploran diferentes lineas de investigación para las GCS futuras de Airbus. Este planificador está basado en algoritmos evolutivos que permite optimizar las trayectorias de búsqueda de varios UAVs para misiones de búsqueda en tiempo mínimo, teniendo en cuenta la información disponible sobre el escenario. Además, describe métodos de ayudan al operador a modelar la incertidumbre sobre la posición y dinámica del objetivo, a partir de la información de la elevación del terreno y de la proporcionada por los servicios de emergencia. Por último, y mediante un caso de uso basado en un escenario realista, se muestra un ejemplo de la metodología propuesta y de los resultados obtenidos con el algoritmo.

Como trabajo futuro se desea extender la metodología de modelado a otros tipos de escenarios, ampliando el número de fuentes de información (por ejemplo usando mapas de ríos o de carreteras). Además, se comprobará el funcionamiento del planificador sobre UAVs de ala rotatoria utilizando un modelo Simulink adecuado.

\section{Agradecimientos}

Este trabajo ha sido financiado por Airbus Defence and Space con el proyecto SAVIER AER-30459.

\section{English summary}

\section{MODELLING AND OPTIMIZING TARGET SEARCH MISSIONS WITH UAVs}
Abstract
Looking for lost targets with one or seve- ral UAVs is a problem with applications ranging from military search missions to 
search and rescue operations after natural disasters. The search time can be minimized by an algorithm that determines the $U A V$ s routes that minimize the expected time of target detection considering the available information and uncertainty associated with each mission and search scenario. Moreover, the suitability of the routes proposed by these algorithms depends both on the used optimization techniques and on the adaptation of the models to the available information and existing uncertainty. Therefore, this article addresses the MTS problem from a global perspective that includes the search scenario modelling phase and the trajectory optimization by means of evolutionary metaheuristics.

Keywords: Minimum Time Search, Planning under Uncertainty, UAVs, Genetic Algorithms

\section{Referencias}

[1] F. Bourgault, T. Furukawa, and H.F. Durrant-Whyte. Optimal Search for a Lost Target in a Bayesian World. Springer Berlin Heidelberg, 2006.

[2] K. Deb. An efficient constraint handling method for genetic algorithms. Computer methods in applied mechanics and engineering, 186(2), 2000.

[3] D. Goldberg. Genetic Algorithms in Search, Optimization and Machine Learning. 1989.

[4] J. Insenser. Open innovation for future rpas ground control system. World ATM Congress, www.worldatmcongress. org/2016-presentations

[5] B.O. Koopman. Search and screening: general principles with historical applications. 1980.

[6] P. Lanillos, E. Besada-Portas, G. Pajares, and J.J. Ruz. Minimum time search for lost targets using cross entropy optimization. In IEEE/RSJ International Conference on Intelligent Robots and Systems, 2012.

[7] P. Lanillos, J. Yañez Zuluaga, J.J. Ruz, and E Besada-Portas. A bayesian approach for constrained multi-agent minimum time search in uncertain dynamic domains. In $G e$ netic and Evolutionary Computation Conference, 2013.

[8] Z. Li, Q. Zhu, and C. Gold. Digital terrain modeling: principles and methodology. 2004.

[9] S. Pérez-Carabaza, J. Bermúdez-Ortega, E. Besada-Portas, J.A. López-Orozco, and J.M. de la Cruz. A multi-UAV minimum time search planner based on ACOR. In Genetic and Evolutionary Computation Conference, 2017.

[10] S. Pérez-Carabaza, E. Besada-Portas, J.A. López-Orozco, and J.M. de la Cruz. Planificador de búsqueda en tiempo mínimo en un sistema de control de RPAS. In XXXVII Jornadas de Automática, 2016.

[11] S. Pérez-Carabaza, E. Besada-Portas, J.A. López-Orozco, and J.M. de la Cruz. A real world multi-UAV evolutionary planner for minimum time target detection. In Genetic and Evolutionary Computation Conference, 2016.

[12] S. Pérez-Carabaza, E. Besada-Portas, J.A. López-Orozco, and G. Pajares. Minimum time search in real-world scenarios using multiple UAVs with onboard orientable cameras. Journal of Sensors, 2019, 2019.

[13] M. Raap, S. Meyer-Nieberg, S. Pickl, and M. Zsifkovits. Aerial vehicle search-path optimization a novel method for emergency operations. Journal of Optimization Theory and Applications, 172(3), 2016.

[14] M. Raap, M. Preuß, and S. Meyer-Nieberg. Moving target search optimization - a literature review. Computers \& Operations Research, 105, 2019.

[15] K.E. Trummel and J.R. Weisinger. The complexity of the optimal searcher path problem. Operations Research, 34(2), 1986.

[16] Y. Yang, A. Minai, and M. Polycarpou. Decentralized cooperative search in UAV's using opportunistic learning. In AIAA Guidance, Navigation, and Control Conference and Exhibit, 2002.

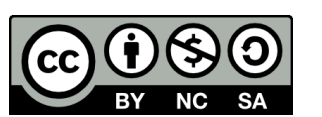
(c) 2019 by the authors. Submitted for possible open access publication under the terms and conditions of the Creative Commons Attribution CC BY-NC-SA 4.0 license (https://creativecommons.org/licenses/by-ncsa/4.0/deed.es). 